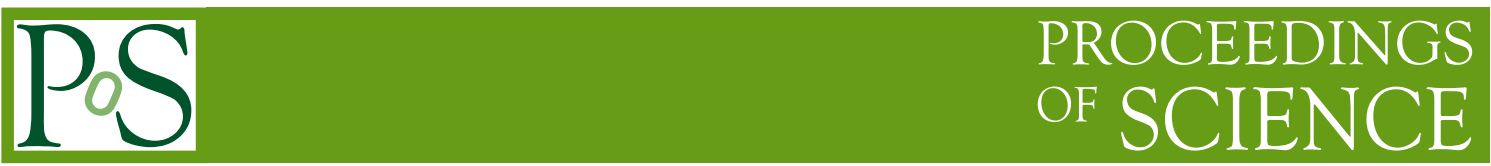

\title{
Database for Nuclear EoS
}

\author{
Chikako Ishizuka* \\ Faculty of Science and Technology, Tokyo University of Science, 2641 Yamazaki, Noda, Chiba, \\ 278-8510 Japan \\ E-mail: Chikakoers.tus.ac.ip
}

Takuma Suda

National Astronomical Observatory of Japan, 2-21-1 Osawa, Mitaka, Tokyo, 181-8588, Japan E-mail: takuma.sudaenao.ac.jp

\section{Hideyuki Suzuki}

Faculty of Science and Technology, Tokyo University of Science, 2641 Yamazaki, Noda, Chiba, 278-8510, Japan

E-mail: suzukihdrs.tus.ac.jp

\section{Akira Ohnishi}

Yukawa Institute for Theoretical Physics, Kyoto University, Kitashirakawa Oiwakecho,

Sakyo-ku, Kyoto 606-8502, Japan

E-mail: ohnishidyukawa.kyoto-u.ac.jp

\section{Kohsuke Sumiyoshi}

Numazu College of Technology, Ooka 3600, Numazu, Shizuoka 410-8501, Japan

E-mail: sumienumazu-ct.ac.ip

It is a long standing problem in nuclear physics and astrophysics to determine the nuclear EoS, which is important in many astrophysical applications, such as simulations of supernova explosions and the constraint of the maximum mass of neutron stars. There are hundreds of published papers dealing with nuclear EoSs, while more than ten nuclear EoS tables are available in tabulated form. In spite of many published EOSs, only a small number of datasets are used in the astrophysical applications. Thus we have constructed a database for nuclear EoSs "EOSDB" which contains information about nuclear theory, HIC experimental analysis, and the observations of NSs relevant to the EoS. The EOSDB is a powerful online tool to investigate the model dependence of a huge number of EoSs, not only for theoretical studies but also for experimental and observational constraints. Users of the EOSDB can compare EoSs, download numerical data, and obtain the details of the method adopted in the original papers.

XII International Symposium on Nuclei in the Cosmos,

August 5-12, 2012

Cairns, Australia

\footnotetext{
* Speaker.
} 


\section{Introduction}

The properties of nuclear equation of state (hereafter EoS) play an important role both in nuclear physics and astrophysics, because the EoS of dense matter is directly related not only to the strong interaction, which is one of the four fundamental interactions of nature, but also to compact object formations such as dynamical evolution of type-II supernovae, neutron star, and black hole formation. However, the properties of EOSs are still to be examined except for nuclear saturation properties. In order to constrain the EoS, the incompressibilities of nuclei and observed neutron star mass have been investigated for the last two decades. In addition to these properties, the symmetry energy of nuclear matter has attracted our attention. Thanks to the development of experimental technique for unstable nuclei, we can obtain information on the symmetry energy by mass formulae, isobaric analogue states, pigmy dipole resonance, heavy ion collisions, and neutron skin thickness. Therefore the interdisciplinary collaborations between nuclear physics, hadron physics, and astrophysics has recently become a more popular way to understand nuclear EoS.

Our investigations of papers published before 2011 have led us to discover a trend in publications regarding EOSs. Hundreds of papers about nuclear EoS have been published in major journals which have been clearly increasing after the discovery of a massive neutron star with $2 M_{\odot}$ " [四]. However, the number of EoSs which we can see in the astrophysical context, is very limited, e.g., polytropic EoSs, well-known Lattimer-Swesty's EoS [0] or Shen's EoS [Q]. There exists a huge number of EoSs which are not restricted to dense matter and contain updated information on nuclear structure or interactions etc. This leads to a very simple but vitally important question; Why astrophysicists do not refer to such various EoSs? That is the motivation of our project: to construct a database for nuclear EoSs. The difficulty in sharing our knowledge on the EoS with other research fields is mainly caused by the diversity of the hadronic phase and the properties of the nuclear force, i.e., an alpha condensation, a liquid-gas phase transition for hot nuclear systems, a nuclear pasta phase, the appearance of hyperons, a pion condensation, a Kaon condensation, a quark-gluon plasma, a hadron quark phase transition and quark phase structures such as color super conductivity, etc.

In general, we use the appropriate models and/or methods for the relevant target. The ab initio calculations, such as lattice QCD and many-body calculations based on a no core shell model, are not always the most reliable in nuclear physics because we cannot exclude the phenomenological factor due to the ambiguity of the strong interaction. Then we should choose a suitable model to treat particular phenomenon. Each research field has a certain consensus on the qualitative behaviour of representative nuclear theoretical models. For example, a relativistic model gives a stiffer EoS than a non-relativistic one, a three-body force makes a stiffer EoS than a only two-body force, substitution of the Lorentz boost correction for the relativity makes an EoS stiffer as well, a quantum hydrodynamic effect makes an EoS stiffer than Fermi-Dirac statistics, and etc. The most important point here is that we should take account of model-dependence of analysed data and theoretical results. The experimental and observational constraints that we know on nuclear EoSs always contain some contributions from theoretical models used. Our aim is to construct a useful tool to analyse and share a particular trends for models quantitatively and qualitatively, and, then to derive the essential information for the EoS. In the following sections we show how our database, the EOSDB, can help to compare and study EoSs. 


\section{Set up}

The EOSDB succeeded the basic structure of the SAGA database [4], [5] which is a database system for observed metal-poor stars. The EOSDB has been operating with MySQL and CGI and the retrieval and data plotting systems were constructed with Perl and JavaScript. We prepared dictionaries for compilation of the EoSs; the list of major journals, the list of basic physics constants which are used in the calculation, the list of classifications for constituents of dense matter, the list of methods, the list of thermodynamical variables, and the list of physical quantities on symmetric energies. By using these dictionaries, we make an entry file for each interaction in each article. In our database, users can check and compare not only the basic EoS properties such as thermodynamical quantities but also the symmetry energy $\mathrm{S}$, its slope $\mathrm{L}$ and the incompressibility $\mathrm{K}$ as a function of baryon densities with various models. Currently, all of our entries are for data basically at $T=0 \mathrm{MeV}$ for symmetric nuclear matter and/or pure neutron matter to focus on the most basic features of hadronic matter and to show differences in the behaviour of each model. As at the end of September 2012, 24 theoretical data have been registered in the EOSDB, and one of them is about constraint based on the experimental data analysis. Recording of nuclear EoSs has been performed using open EoS tables and by using a graph read system GSYS distributed by JCPRG [3]. It is to be noted that nuclear physicists dare not make EoS tables. That is why the tabulated EoSs are only the tip of an iceberg. Most of data in the EOSDB has been compiled by GSYS at present and such data may cause systematic errors because the work relies on manual operation.

\section{EOSDB}

The EOSDB consists of a database for nuclear EoSs and an online retrieval system working with query options. By using the EOSDB, we can compare various EoSs online and download plotted data. The data can be saved as png, eps, pdf and text format. In addition, the EOSDB provides the following dataset for each entry as well as data tables:

1. Bibliographic information

2. Manual for EoS table in case the tabulated data exists

3. Physics constants used in each EoS

4. Assumed constituents and conditions

5. Theoretical/Experimental/Observational methods to derive each EoS and its strong/weak point and comments.

6. Saturation density

7. Saturation energy

8. Symmetry Energy properties

9. Maximum cold neutron star mass (if it can be calculated)

The following are two examples to show how the EOSDB works. Although we concentrate on the symmetry energy for simplicity, we can investigate the other quantities in the selected data set. (i) Figure 1 compares the symmetry energy as a function of baryon density from different approaches in order to show the reproducibility of a figure in the reference [ [8] by using the EOSDB. In this figure, we show a wider $\mathrm{x}$ range than the original data to plot whole data points recorded in our database. Users can reproduce the original figure by entering the values of the $\mathrm{x}$ range 
in the web form. The web interface allows users to switch linear or log scale for horizontal and vertical axes independently. Four different points captioned as "A. Akmal (1998) " are data points taken from tables shown in [ [ 9 ]. The original data is shown by tables of energy as a function of baryon density. We derived symmetry energy by the difference between the energy of pure neutron matter and that of symmetric nuclear matter. These four data points correspond to symmetry energy by using an ab initio variational method with a two-baryon interaction AV18, AV18 + the Lorentz boost, AV18 and a three-body force, AV18 + the three-body force + the Lorentz boost, respectively. Data described as "E. N. E. Dalen (2004)" was recorded with GSYS. The original data is shown in Fig.2 of [5] as a subtracted T -matrix representation. Data labelled "S. Typel (1999)" was recorded from their Fig.5 in [ [8]. These data are based on a phenomenological density-dependent Relativistic Mean Field (RMF) functional DD-TW [四]. Data with error bars, "Niksic (2002)" were recorded with GSYS from Fig.5 in [ $[8]$. We represent the constraint on the symmetry energy with error bars, while it is shown as a shaded area in the original figure.

(ii) Figure 2 also shows the symmetry energy as a function of baryon density in order to propose a further possible usage of the EOSDB beyond the reproduction in Fig.1. We compare recently published three RMF models compiled in the EOSDB with the constraint shown in Fig.1. At present, no one knows which model and method is suitable to describe the EoS. That is why we choose the models from the perspective of an affinity with our previous work [[13] on the EoS tables with RMF. The EOSDB makes it possible to compare various EoS models by a specific theoretical framework and can derive each characteristic caused by the detailed difference between models. Data labelled "Shen (2011)" [ए]] is basically the same EoS with their previous EoS table under the saturation density, which is widely used in the astrophysical hydrodynamical calculations as well as he Lattimer\&Swesty EoS [D]. But their current EoS contains $\Lambda$ hyperons by using the RMF parameter set TM1. The data points of "Miyatsu (2012)" []], which were calculated by a relativistic Hartree-Fock calculation with a quark-meson coupling model, were recorded from EoS tables by private communication although they are not officially published as a tabulated format. This model, including the full baryon octet, can support a massive neutron star, which is consistent with the Shapiro delay observation [U] because of the Fock term contribution, especially tensor coupling. In "Hempel2010" [[2], nucleons are calculated by the RMF model with the parameter set TMA. An ensemble of nuclei and interacting nucleons is treated by a statistical model, which is consistent with experimental mass data and nuclear structure calculations, included Coulomb and excluded volume effects. This data is subject to limited access. Thus, in the EOSDB, we use their table data by private communication.

Users of the EOSDB are cautioned when they use it. It is not meaningful to compare the data based on different constituents or different assumptions on physical conditions, such as $\beta$ equilibrium. In addition, it is our fundamental policy to keep the consistency between the data in the EOSDB and the original reference. This is demonstrated by the fact that we do not refer to our EoS tables with full baryon octet [113] based on RMF parameter set TM1.

\section{Summary and future prospects}

We have constructed a database for nuclear EoS and its web platform the EOSDB by sharing the modules of the SAGA database that is a database of known extremely metal-poor stars. The 


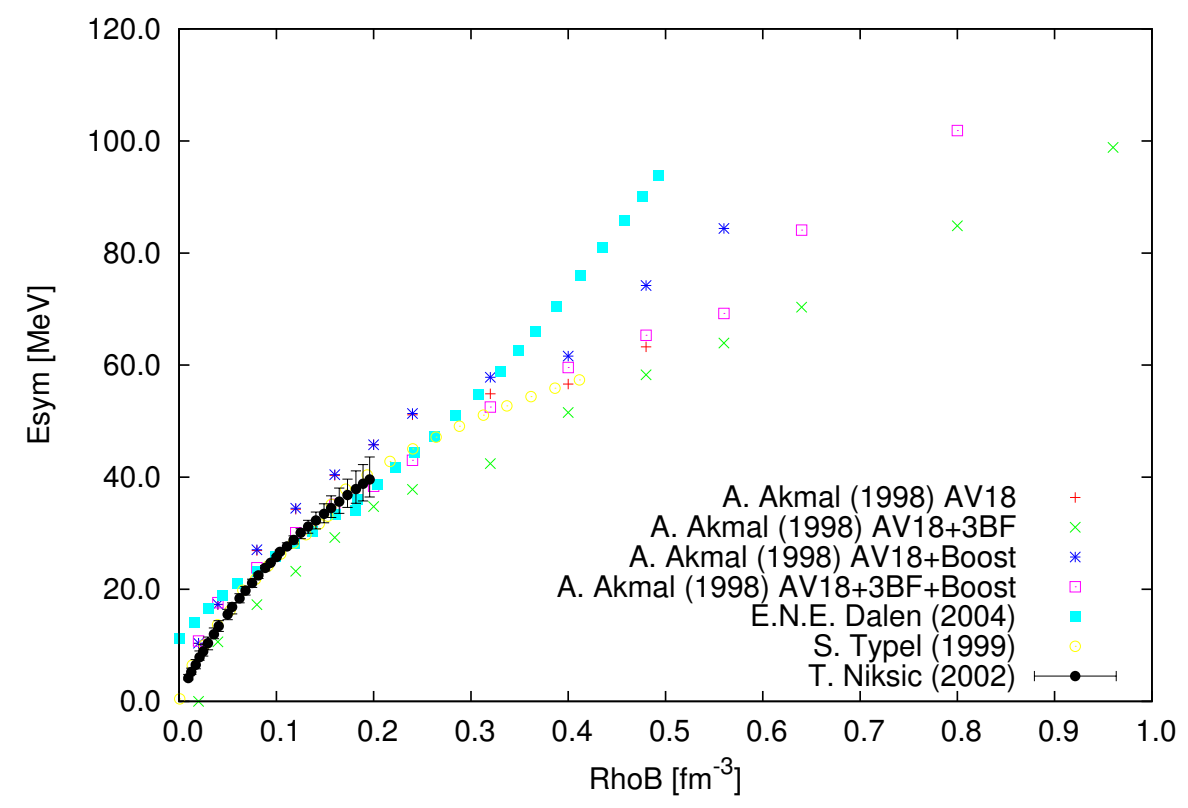

Figure 1: Figure made by the EOSDB, saved online as a eps file and downloaded. Symmetry energy as a function density similar to the Fig.5 in Fuchs et al. 2006 [] $]$. The plotted x range is wider than that of the reference [ [ ] which is less than $0.2\left[\mathrm{fm}^{-3}\right]$.

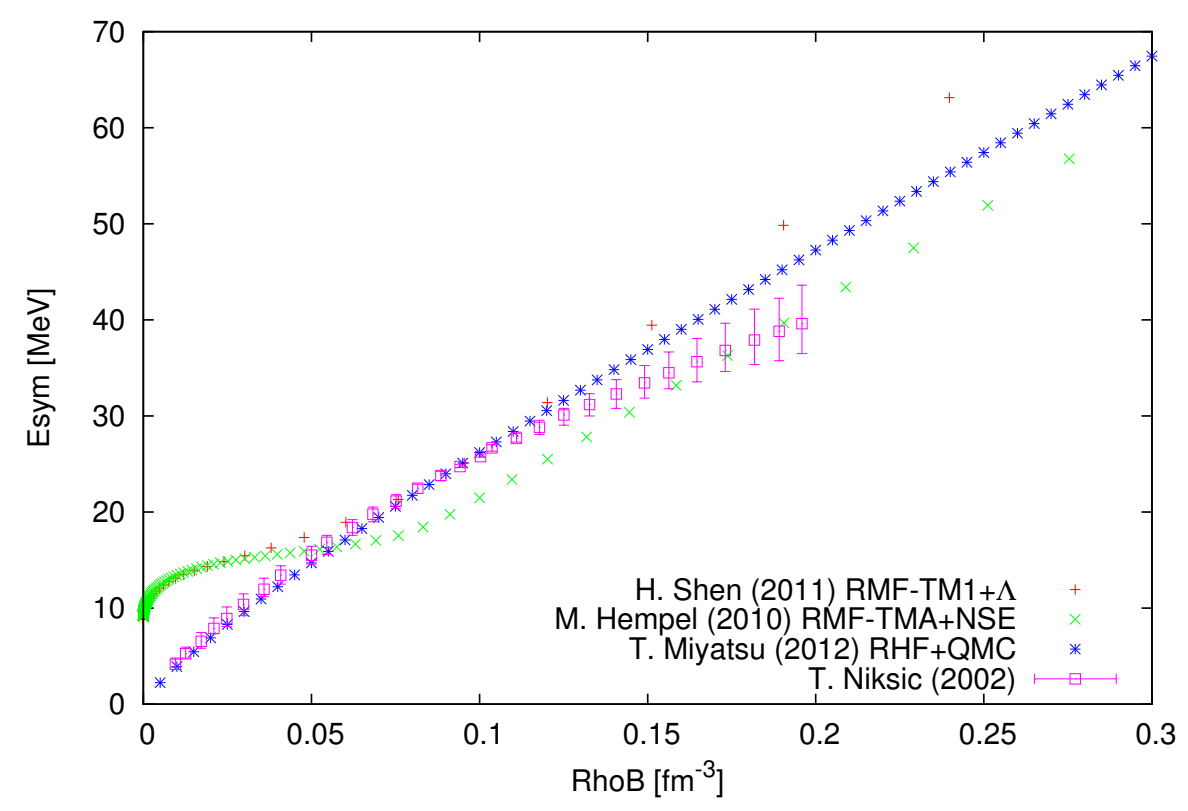

Figure 2: Figure made by the EOSDB, saved online as a eps file and downloaded. Symmetry energy of

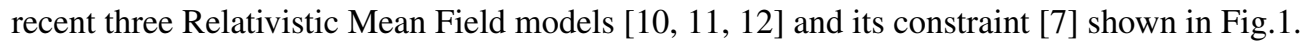


beta-version of the online tools for the EOSDB is available if one of the authors are contacted. The database is very helpful in easily knowing the behaviour of EoSs and in trying data mining with the EoSs. With the use of the EOSDB, we will report (in a forthcoming paper) a benchmark test for various EoSs in the following formats: (1) Thermodynamical data set for symmetric/pure neutron matter, (2) Saturation properties, (3) Symmetry energy propertes as a function of baryon density, (4) Model and Interactions, and (5) High reliability circumstance of each model, e.g. below the saturation density. In this paper, we showed that the EOSDB is equipped with various functions which will be required when we make a benchmark test for nuclear EoSs and share the information with others. In the future updates, we will add the query options with "model/method" on the retrieval function in order to elucidate the model dependence of EoSs and the contribution of background physics conditions to EoSs, qualitatively and quantitatively. We hope the EOSDB will be a helpful tool to investigate a super-phenomenological EoS.

\section{Acknowledgments}

The authors thank to Matthias Hempel and Tsuyoshi Miyatsu for kindly providing data of their theoretical models. CI is grateful to Sasagawa Grants for Scientific Fellows of the Japan Science Society, F12-208, for their financial support to attend the conference.

\section{References}

[1] P. B. Demorest, T. Pennucci, S. M. Ransom, M. S. E. Roberts and J. W. T. Hessels, Nature 467, 1081 (2010).

[2] J. M. Lattimer and D. F. Swesty, Nucl. Phys. A 535, 331 (1991).

[3] GSYS, http://www.jcprg.org/gsys/gsys-j.html

[4] A. Akmal, V. R. Pandharipande, D. G. Ravenhall, Phys. Rev. C 58, 1804 (1998).

[5] E. van Dalen, C. Fuchs, A. Faessler, Nucl. Phys. A 744, 227 (1999).

[6] S. Typel, H. H. Wolter, Nucl. Phys. A 656, 331 (1999).

[7] T. Niksic, D. Veretenar, P. Ring, Phys. Rev. C 66, 064302 (2002).

[8] C. Fuchs and H.H. Wolter, Eur. Phys. J. A 30, 5 (2006).

[9] H. Shen, H. Toki, K. Oyamatsu, and K. Sumiyoshi, Nucl. Phys. A 637, 435 (1998).

[10] H. Shen, H. Toki, K. Oyamatsu, and K. Sumiyoshi, Astrophys. J. Suppli. 197, Num.2, 20 (2011).

[11] T. Miyatsu, T. Katayama, K. Saito, Phys. Lett B 709242 (2012).

[12] M. Hempel, J. Schaffner-Bielich, Nucl. Phys. A 837210 (2010).

[13] C. Ishizuka, A. Ohnishi, K. Tsubakihara, K. Sumiyoshi, S. Yamada, J. of Phys. G 35, 08521 (2008).

[14] T. Suda, Y. Katsuta, S. Yamada, T. Suwa, C. Ishizuka, Y. Komiya, K. Sorai, M. Aikawa, and M.Y. Fujimoto, Publi. of Astron. Soc. of Japan, 60, 1159, (2008).

[15] T. Suda, S. Yamada, Y. Katsuta, Y. Komiya, C. Ishizuka, W. Aoki, and M.Y. Fujimoto, Mon. Not. of Roy. Astron. Soc., 412, Issue 2, 843 (2011). 